PROCEEDINGS OF THE

AMERICAN MATHEMATICAL SOCIETY

Volume 131, Number 11, Pages 3423-3430

S 0002-9939(03)07023-0

Article electronically published on March 25, 2003

\title{
ON A PROBLEM OF TURÁN ABOUT POSITIVE DEFINITE FUNCTIONS
}

\author{
MIHAIL N. KOLOUNTZAKIS AND SZILÁRD GY. RÉVÉSZ
}

(Communicated by Andreas Seeger)

\begin{abstract}
We study the following question posed by Turán. Suppose $\Omega$ is a convex body in Euclidean space $\mathbb{R}^{d}$ which is symmetric in $\Omega$ and with value 1 at the origin; which one has the largest integral? It is probably the case that the extremal function is the indicator of the half-body convolved with itself and properly scaled, but this has been proved only for a small class of domains so far. We add to this class of known Turán domains the class of all spectral convex domains. These are all convex domains which have an orthogonal basis of exponentials $e_{\lambda}(x)=\exp 2 \pi i\langle\lambda x\rangle, \lambda \in \mathbb{R}^{d}$. As a corollary we obtain that all convex domains which tile space by translation are Turán domains.

We also give a new proof that the Euclidean ball is a Turán domain.
\end{abstract}

\section{A problem of Turán}

The following question is attributed to Turán (and Stechkin [1]):

(Turán) Let $\Omega$ be a convex domain in $\mathbb{R}^{d}$ which is symmetric with respect to 0 . What is the maximum of $\int f$ for all $f$ supported in $\Omega$ which are positive definite (their Fourier Transform is nonnegative) and have $f(0)=1$ ?

See [2, 5, 13] for the history of the problem.

Definition 1 (Turán domains). A symmetric convex domain $\Omega$ is called a Turán domain if the maximum of $\int f$ over all positive definite functions supported in $\Omega$ and with $f(0)=1$ is achieved by the function

$$
f=\frac{2^{d}}{|\Omega|} \chi_{\frac{1}{2} \Omega} * \chi_{\frac{1}{2} \Omega}
$$

Otherwise it is called an anti-Turán domain.

Received by the editors May 22, 2002.

2000 Mathematics Subject Classification. Primary 42B10; Secondary 26D15, 52C22, 42A82, $42 \mathrm{~A} 05$.

Key words and phrases. Fourier transform, positive definite functions, Turán's extremal problem, convex symmetric domains, tiling of space, lattice tiling, spectral domains.

The first author was supported in part by INTAS grant, project 99-01080.

The second author was supported in part by the Hungarian National Foundation for Scientific Research, Grant \# T034531 and T032872. 
The function $f$ in the above definition is clearly a legitimate function, as its Fourier Transform (FT) is

$$
\widehat{f}=\frac{2^{d}}{|\Omega|}\left|\widehat{\chi_{\frac{1}{2} \Omega}}\right|^{2}
$$

and $f(0)=1$. The integral of $f$ in this case is $2^{-d}|\Omega|$.

Anti-Turán domains are not known to exist.

We cite the recent papers by Arestov and Berdysheva [2] and by Gorbachev [5] 11 In [2] it is proved that the regular hexagon in the plane is a Turán domain and in [5] that the Euclidean ball in $d$-dimensional space is also one. We find it very likely that there are no anti-Turán domains.

Remark. This problem is only interesting for symmetric domains, as the support of any positive definite function is always a symmetric set. The convexity assumption on the domain also cannot be removed if the function $f$ in (1) is to be supported in $\Omega$.

\section{A problem of Fuglede}

Our interest in this problem of Turán arose in connection with a conjecture of Fuglede which we now describe.

Definition 2 (Translational tiles). A measurable set $\Omega \subseteq \mathbb{R}^{d}$ is a translational tile if there exists a set $\Lambda \subseteq \mathbb{R}^{d}$ such that almost all (Lebesgue) points in $\mathbb{R}^{d}$ belong to exactly one of the translates

$$
\Omega+\lambda, \quad \lambda \in \Lambda .
$$

We denote this condition by $\Omega+\Lambda=\mathbb{R}^{d}$.

If $f \in L^{1}\left(\mathbb{R}^{d}\right)$ is nonnegative we say that $f$ tiles with $\Lambda$ at level $\ell$ if

$$
\sum_{\lambda \in \Lambda} f(x-\lambda)=\ell, \text { a.e. } x .
$$

We denote this latter condition by $f+\Lambda=\ell \mathbb{R}^{d}$.

In any tiling the translation set has some properties of density which hold uniformly in space.

Definition 3 (Uniform density). A multiset $\Lambda \subseteq \mathbb{R}^{d}$ has (uniform) density $\rho$ if

$$
\lim _{R \rightarrow \infty} \frac{\#\left(\Lambda \cap B_{R}(x)\right)}{\left|B_{R}(x)\right|} \rightarrow \rho
$$

uniformly in $x \in \mathbb{R}^{d}$. We write $\rho=\operatorname{dens} \Lambda$.

We say that $\Lambda$ has (uniformly) bounded density if the fraction above is bounded by a constant $\rho$ uniformly for $x \in \mathbb{R}$ and $R>1$. We then say that $\Lambda$ has density (uniformly) bounded by $\rho$.

Remark. It is not hard to prove (see for example [9], Lemma 2.3, where it is proved in dimension one - the proof extends verbatim to higher dimension) that in any tiling $f+\Lambda=\ell \mathbb{R}^{d}$ the set $\Lambda$ has density $\ell / \int f$.

\footnotetext{
${ }^{1}$ We thank Professor Arestov for these references.
} 
Let $\Omega$ be a measurable subset of $\mathbb{R}^{d}$ and $\Lambda$ be a discrete subset of $\mathbb{R}^{d}$. We write

$$
\begin{aligned}
e_{\lambda}(x) & =\exp 2 \pi i\langle\lambda, x\rangle \quad\left(x \in \mathbb{R}^{d}\right), \\
E_{\Lambda} & =\left\{e_{\lambda}: \lambda \in \Lambda\right\} \subset L^{2}(\Omega) .
\end{aligned}
$$

The inner product and norm on $L^{2}(\Omega)$ are

$$
\langle f, g\rangle_{\Omega}=\int_{\Omega} f \bar{g} \text { and }\|f\|_{\Omega}^{2}=\int_{\Omega}|f|^{2} .
$$

Definition 4 (Spectrum of a domain, spectral pairs). The pair $(\Omega, \Lambda)$ is called a spectral pair if $E_{\Lambda}$ is an orthogonal basis for $L^{2}(\Omega)$. A set $\Omega$ will be called spectral if there is $\Lambda \subset \mathbb{R}^{d}$ such that $(\Omega, \Lambda)$ is a spectral pair. The set $\Lambda$ is then called a spectrum of $\Omega$.

Example. If $Q_{d}=(-1 / 2,1 / 2)^{d}$ is the cube of unit volume in $\mathbb{R}^{d}$, then $\left(Q_{d}, \mathbb{Z}^{d}\right)$ is a spectral pair, as is well known by the ordinary $L^{2}$ theory of multiple Fourier series.

The following conjecture is still unresolved, in all dimensions and both directions.

Conjecture 1 (Fuglede [4]). Let $\Omega \subset \mathbb{R}^{d}$ be a bounded open set. Then $\Omega$ is spectral if and only if there exists $L \subset \mathbb{R}^{d}$ such that $\Omega+L=\mathbb{R}^{d}$ is a tiling.

Note that in Fuglede's Conjecture no relation is claimed between the translation set $L$ and the spectrum $\Lambda$. This conjecture is still open for all dimensions. However (see for example [4, 10]), the lattice case of this conjecture is easy to show. In the following result the dual lattice $\Lambda^{*}$ of a lattice $\Lambda$ is defined as usual by

$$
\Lambda^{*}=\left\{x \in \mathbb{R}^{d}: \forall \lambda \in \Lambda \quad\langle x, \lambda\rangle \in \mathbb{Z}\right\} .
$$

Theorem A (Fuglede [4). The bounded, open domain $\Omega$ admits translational tilings by a lattice $\Lambda$ if and only if $E_{\Lambda^{*}}$ is an orthogonal basis for $L^{2}(\Omega)$.

\section{Connecting the problems of Turán And Fuglede}

Our main result is the following.

Theorem 1. Let $\Omega \subseteq \mathbb{R}^{d}$ be a symmetric convex domain. If $\Omega$ is spectral, then it has to be a Turán domain as well.

It follows that convex spectral domains are Turán domains. Indeed, convex spectral domains are necessarily symmetric according to the result in [10], and so the above Theorem 1 applies.

Corollary 1. Suppose the symmetric convex domain $\Omega \subseteq \mathbb{R}^{d}$ is a translational tile. Then it is a Turán domain.

Proof of Corollary 1 We start with the following result which claims that every convex tile is also a lattice tile.

Theorem B (Venkov [15] and McMullen [14]). Suppose that a convex body K tiles space by translation. Then it is necessarily a symmetric polytope and there is a lattice $L$ such that

$$
K+L=\mathbb{R}^{d}
$$


A complete characterization of the tiling polytopes is also among the conclusions of the Venkov-McMullen Theorem but we do not need it here and choose not to give the full statement, as it would require some more definitions.

So, if a convex domain is a tile, it is also a lattice tile, hence spectral by Theorem A, and as such it is Turán, by Theorem 1

Remarks. 1. If one wants to avoid using the Venkov-McMullen Theorem in the proof of Corollary 1 one should enhance the assumption of Corollary 1 to state that $\Omega$ is a lattice tile.

2. We have just received a recent preprint [3] of Arestov and Berdysheva, in which they prove Corollary 1 without going through spectral domains.

The result of 2 about the hexagon being a Turán domain is thus a special case of our Corollary [1, but not the result in [5] about the ball being Turán. The ball [6], and essentially every smooth convex body [7], is known not to be spectral, in accordance with Conjecture 1

Fuglede's Conjecture for convex domains is still open except for dimension $d=2$, in which case it was recently answered in the affirmative; see [8]. Thus our Theorem 11 conceivably (though not very likely) applies to a wider class of convex domains than just convex tiles, dealt with in Corollary 1 .

Proof of Theorem 1] The proof of our main theorem relies on Fourier theoretic characterizations of translational tiling [10]. Without loss of generality let us assume from now on that $\Omega$ has volume 1 .

Let $\Omega$ have spectrum $\Lambda \subseteq \mathbb{R}^{d}$. This is equivalent to the following (see [10]):

$$
\sum_{\lambda \in \Lambda}|\widehat{\chi \Omega}|^{2}(x-\lambda)=1, \quad \text { for a.e. } x \in \mathbb{R}^{d} .
$$

That is, $|\widehat{\chi \Omega}|^{2}$ tiles $\mathbb{R}^{d}$ with translation set $\Lambda$ at level 1 , i.e. $|\widehat{\chi \Omega}|^{2}+\Lambda=\mathbb{R}^{d}$. It follows (see the Remark after Definition [3) that dens $\Lambda=1 /|\Omega|=1$.

For any given $\Lambda \subset \mathbb{R}^{d}$ with bounded density (see Definition 3) we denote by $\delta_{\Lambda}$ the (infinite) measure $\sum_{\lambda \in \Lambda} \delta_{\lambda}$. This is a tempered distribution, as the total mass in a ball of radius $R$ grows polynomially with $R$, and therefore we can speak of its FT.

We shall use the following result from [10].

Lemma 1 (Kolountzakis [10]). Suppose that $f \geq 0$ is not identically 0 , that $f \in$ $L^{1}\left(\mathbb{R}^{d}\right), \widehat{f} \geq 0$, has compact support and $\Lambda \subset \mathbb{R}^{d}$. If $f+\Lambda$ is a tiling, then

$$
\operatorname{supp} \widehat{\delta_{\Lambda}} \subseteq\left\{x \in \mathbb{R}^{d}: \widehat{f}(x)=0\right\} \cup\{0\}
$$

When applied to our case, $f=\left|\widehat{\chi_{\Omega}}\right|^{2}$ and $\widehat{f}=\chi_{\Omega} * \chi_{\Omega}$, and it follows that

$$
\operatorname{supp} \widehat{\delta_{\Lambda}} \subseteq\{0\} \cup(\Omega-\Omega)^{c}=\{0\} \cup(2 \Omega)^{c} .
$$

The necessary support condition (3) in Lemma 1 cannot by itself guarantee that $f$ tiles with $\Lambda$. The reason is that a tempered distribution, such as $\widehat{\delta_{\Lambda}}$, which is supported in the zero-set of a function, is not necessarily killed when multiplied by that function. One has to know some extra information about the order of the 
distribution ("what order derivatives it involves") versus the degree of vanishing of the function on the support of the distribution 2

In the following partial converse to Lemma 1] (see [10]), this problem is solved as the separation of the supports guarantees infinite order of vanishing of $f$.

Lemma 2 (Kolountzakis [10]). Suppose that $g \in L^{1}\left(\mathbb{R}^{d}\right)$, and that $\Lambda \subset \mathbb{R}^{d}$ has uniformly bounded density. Suppose also that $O \subset \mathbb{R}^{d}$ is open, that

$$
\widehat{g}(0)=\int g \neq 0,
$$

and that for some $\delta>0$,

$$
\operatorname{supp} \widehat{\delta_{\Lambda}} \backslash\{0\} \subseteq O \text { and } O+B_{\delta}(0) \subseteq\{\widehat{g}=0\}
$$

Then $g+\Lambda$ is a tiling at level $\widehat{g}(0) \cdot \widehat{\delta_{\Lambda}}(\{0\})$.

The conclusion of Lemma 2 demands some explanation. Conditions (5) and (6) imply that in a neighborhood of 0 the tempered distribution $\widehat{\delta_{\Lambda}}$ is supported at 0 only. That is because $\widehat{g}$ is continuous and, since $\widehat{g}(0) \neq 0$, it does not vanish in some neighborhood of 0 . It then follows that, near $0, \widehat{\delta_{\Lambda}}$ is not only a tempered distribution but a measure, that is, it is just a point mass at 0 (see 9], Theorem 5.1 , Step 1, for the proof in dimension 1, which works in any dimension). For this reason it makes sense to write $\widehat{\delta_{\Lambda}}(\{0\})$ for that point mass.

From Lemma 3 below, it follows that the value of this constant is precisely the density of $\Lambda$, if such a density exists.

Lemma 3 (Kolountzakis 11]). Suppose that $\Lambda \in \mathbb{R}^{d}$ is a multiset with density $\rho$, $\delta_{\Lambda}=\sum_{\lambda \in \Lambda} \delta_{\lambda}$, and that $\widehat{\delta_{\Lambda}}$ is a measure in a neighborhood of 0 . Then $\widehat{\delta_{\Lambda}}(\{0\})=\rho$.

Conclusion of the proof of Theorem 11. If $\Omega$ is of anti-Turán type, then there exists a positive definite function $F$ supported in $\Omega$ with $F(0)=1$ and $\int F>2^{-d}$.

Now define

$$
\widehat{G}(x)=F\left(\frac{1+\epsilon}{2} x\right),
$$

where $\epsilon>0$ is to be taken so small that we have $\int \widehat{G}>1$. It follows that supp $\widehat{G} \subseteq$ $(1-\theta) 2 \Omega$, for some $\theta>0$. The function $\widehat{G}$ is also positive definite.

Because of (4) we can now write

$$
\operatorname{supp} \widehat{\delta_{\Lambda}} \subseteq\{0\} \cup\{\widehat{G}=0\}
$$

Here we aim to apply Lemma 2, with $O=(\overline{(2-\theta) \Omega})^{c}$. First let us observe the following simple fact.

Lemma 4. Let $0 \leq \alpha \leq \beta$. Then for any bounded convex domain $\Omega$ with $0 \in \Omega$ we have $\operatorname{dist}\left\{\alpha \Omega,(\beta \Omega)^{c}\right\}=r(\beta-\alpha)$, where $r:=\operatorname{dist}\left\{0, \Omega^{c}\right\}=\max \left\{\rho: B_{\rho} \subseteq \Omega\right\}$ is the inradius of $\Omega$.

\footnotetext{
${ }^{2} \mathrm{An}$ important special case is when one knows the distribution to be a measure, as is the case when $\Lambda$ is either a lattice or fully periodic. In that case any vanishing of the function will do and the implication in Lemma 1 can essentially be reversed.

3 Condition (5) was mistakenly omitted from [10]. This does not affect the validity of what is proved in [10], as condition (5) is easily seen to hold in the specific application.
} 


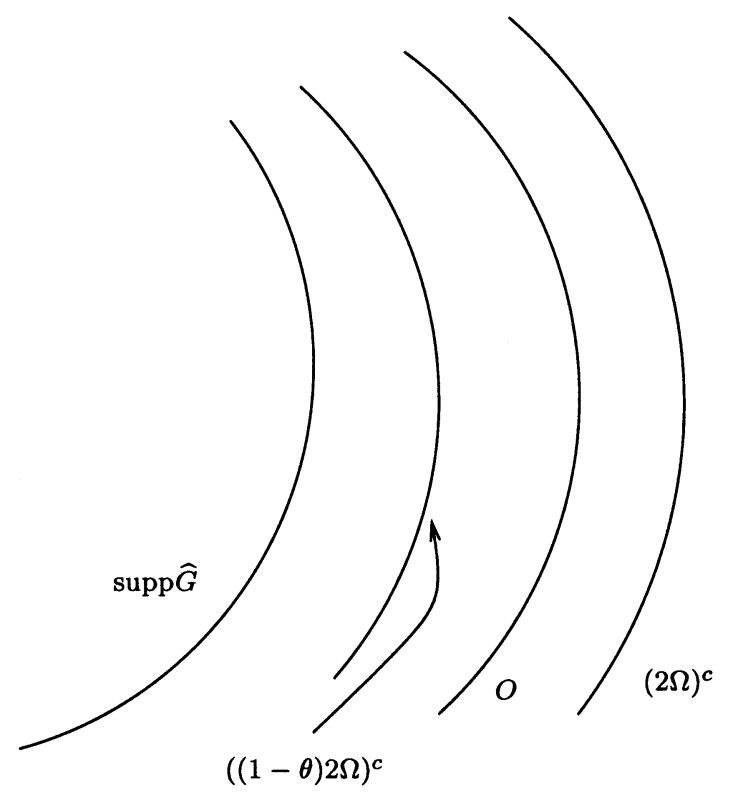

Figure 1. The domains used in the proof of Theorem 1

Proof. First, if $a \in \alpha \Omega, \rho<r$ and $y \in(\beta-\alpha) B_{\rho}$, then we have $a+y \in \alpha \Omega+$ $(\beta-\alpha) B_{\rho} \subset \alpha \Omega+(\beta-\alpha) \Omega \subset \beta \Omega$ by the convexity of $\Omega$. Thus dist $\left\{a,(\beta \Omega)^{c}\right\} \geq$ $(\beta-\alpha) \rho$ and dist $\left\{\alpha \Omega,(\beta \Omega)^{c}\right\} \geq(\beta-\alpha) r$. On the other hand let $x \in \partial \Omega \cap \partial B_{r}$. Then $\operatorname{dist}\left\{\alpha \Omega,(\beta \Omega)^{c}\right\} \leq \operatorname{dist}\{\alpha x, \beta x\}=(\beta-\alpha) r$.

Thus we have $\operatorname{dist}\{O,(1-\theta) 2 \Omega\}=\theta r$ by the above lemma, hence for any $\delta<\theta r$ we have $O+B_{\delta} \subset((1-\theta) 2 \Omega)^{c}$. On the other hand, $\operatorname{dist}\left\{(2-\theta) \Omega,(2 \Omega)^{c}\right\}=\theta r>0$ again by the above lemma and thus $(2 \Omega)^{c} \subset(\overline{(2-\theta) \Omega})^{c}=O$.

That is, by (4), supp $\widehat{\delta_{\Lambda}} \backslash\{0\} \subseteq(2 \Omega)^{c} \subset O$. With these condition (6) is seen to be fulfilled by $g=G$, as $\{\widehat{g}=0\}=\{\widehat{G}=0\} \supseteq((1-\theta) 2 \Omega)^{c}$ since supp $\widehat{G} \subseteq(1-\theta) 2 \Omega$.

Note that (5) is also satisfied here as $\widehat{g}(0)=\widehat{G}(0)=F(0)=1$ by the definition of $\widehat{G}$. Thus Lemma 2 can be applied and we find that $G$ tiles $\mathbb{R}^{d}$ with translation set $\Lambda$ at level $\widehat{g}(0) \widehat{\delta_{\Lambda}}\{(0)\}=\widehat{G}(0)$ dens $\Lambda$ by Lemma 3 . Here $\widehat{G}(0)=1$ and also dens $\Lambda=1$ in view of the considerations following formula (2). Thus the level of tiling by $G$ and $\Lambda$ is found to be 1 . However, $G(0)=\int \widehat{G}>1$, hence the continuous nonnegative function $G$ cannot tile $\mathbb{R}^{d}$ at level 1 . This contradiction proves that there is no function $F$ with the given properties as supposed at the outset. That is, $\Omega$ is a Turán domain.

\section{ANother Proof that the BALl is a TurÁN DOMAin}

Here we give a new proof, rather different from that in [5], that the ball is a Turán domain. Let $B$ denote the unit ball in $\mathbb{R}^{d}$. To say that the ball is Turán is to prove the inequality

$$
\int f \leq 2^{-d}|B| f(0)
$$


for every positive definite $f$ supported in $B$. By an easy approximation argument it is enough to prove (7) under the extra assumption that $f$ is smooth. Noticing further that both sides of the inequality are linear functionals of $f$ invariant under rotation, we can assume that $f$ is radial by examining the spherical average of $f$,

$$
\int f(T x) d T
$$

the integral being over $T \in O_{n}$, the group of all orthogonal transformations equipped with Haar measure.

The key ingredient in the proof is the following result.

Theorem C (Rudin [12]). Suppose $f$ is a radial smooth positive definite function with support in the ball $B$. Then $f$ can be written as a uniformly convergent series

$$
f=\sum_{k=1}^{\infty} f_{k} * \widetilde{f}_{k}, \quad\left(\widetilde{f}_{k}(x)=\overline{f_{k}(-x)}\right)
$$

with $f_{k}$ being smooth and supported in the half ball $(1 / 2) B$.

Notice that for any integrable function $g$ with support in the compact set $K$ we have for $f=g * \widetilde{g}$

$$
\begin{aligned}
\int f & =\left|\int g\right|^{2} \\
& \leq \int|g|^{2}|K| \quad \text { (Cauchy-Schwartz) } \\
& \leq \int|g|^{2} \cdot 2^{-d}|K-K| \quad \text { (Brunn-Minkowski) } \\
& =f(0) \cdot 2^{-d}|K-K|
\end{aligned}
$$

Taking $K=\overline{(1 / 2) B}$ we obtain for every $f_{k}$ in (8),

$$
\int f_{k} * \widetilde{f}_{k} \leq 2^{-d}|B|\left(f_{k} * \widetilde{f}_{k}\right)(0) .
$$

Summing the series we have (7) for $f$.

\section{ACKNOWLEDGEMENT} lem.

We thank Professor V. Arestov for acquainting us with the history of the prob-

\section{REFERENCES}

[1] N.N. Andreev, personal communication.

[2] V.V. Arestov and E.E. Berdysheva, Turán's problem for positive definite functions with supports in a hexagon, Proc. Steklov Inst. Math., Suppl. 1, 2001, pp. S20-S29.

[3] V.V. Arestov and E.E. Berdysheva, The Turán problem for a class of polytopes, East J. Approx. 8 (2002), 381-388.

[4] B. Fuglede, Commuting self-adjoint partial differential operators and a group theoretic problem, J. Funct. Anal. 16 (1974), 101-121. MR 57:10500

[5] D.V. Gorbachev, An extremal problem for periodic functions with supports in the ball, Math. Notes 69 (2001), 3, 313-319. MR 2002e:42006

[6] A. Iosevich, N. Katz and S. Pedersen, Fourier bases and a distance problem of Erdős, Math. Res. Lett. 6 (1999), no. 2, 251-255. MR 2000j:42013 
[7] A. Iosevich, N. Katz and T. Tao, Convex bodies with a point of curvature do not admit exponential bases, Amer. J. Math. 123 (2001), 115-120. MR 2002g:42011

[8] A. Iosevich, N. Katz and T. Tao, Fuglede's conjecture for convex bodies in the plane, Math. Res. Lett., to appear.

[9] M.N. Kolountzakis and J.C. Lagarias, Structure of tilings of the line by a function, Duke Math. J. 82 (1996), 3, 653-678. MR 97d:11124

[10] M.N. Kolountzakis, Non-symmetric convex domains have no basis of exponentials, Illinois J. Math. 44 (2000), 3, 542-550. MR 2001h:52019

[11] M.N. Kolountzakis, On the structure of multiple translational tilings by polygonal regions, Discr. Comp. Geom. 23 (2000), 537-553. MR 2001c:52025

[12] W. Rudin, An extension theorem for positive definite functions, Duke Math. J. 37 (1970), 49-53. MR 40:7722

[13] S.B. Stechkin, An extremal problem for trigonometric series with nonnegative coefficients, Acta Math Acad. Sci. Hung. 23 (1972), 3-4, pp 289-291 (Russian).

[14] P. McMullen, Convex bodies which tile space by translation, Mathematika 27 (1980), 113-121. MR 82c:52016 acknowledgement of priority MR 83f:52008

[15] B.A. Venkov, On a class of Euclidean polyhedra, Vestnik Leningrad Univ. Ser. Math. Fiz. Him. 9 (1954), 11-31 (Russian). MR 20:1302

Department of Mathematics, University of Crete, Knossos Ave., 71409 Iraklio, Greece

E-mail address: kolount@member.ams.org

Alfréd Rényi Institute of Mathematics, Hungarian Academy of Sciences, 1364 BuDAPEST, HUNGARY

E-mail address: revesz@renyi.hu 